Report no. WD 88-09

Nonlinear free vibrations of

coupled spans of suspended cables

S.W. Rienstra

July 1988

Wiskundige Dienstverlening

Faculteit der Wiskunde en

Natuurwetenschappen

Katholieke Universiteit

Toernooiveld

6525 ED Nijmegen

Contribution to the mini-symposium "Flow induced vibrations of overhead transmission lines", ECMI-Meeting August 28-31, 1988, Glasgow, Scotland.

Proceedings to be published by B.G. Teubner, Stuttgart, W.-Germany. 


\title{
NON-LINEAR FREE VIBRATIONS \\ OF COUPLED SPANS OF SUSPENDED CABLES
}

\author{
S.IV. Rienstra
}

\section{Summary}

The problem of free in-plane nonlinear nearly harmonic vibrations of elastic suspended cables is investigated, with particular emphasis on the configuration of multiple spans, coupled via suspension strings, which is relevant in the context of overhead transmission lines. A systematic asymptotic theory is developed, for a suitable set of small parameters based on a shallow geometry and the presence of only transversal waves. The finally obtained reduced set of equations is solved by a variant of the Lindstedt-Poincaré technique. The (non-trivial) solutions for multiple spans appear to be gravity waves, considerably different from the elasto-gravity waves in the symmetric single span configuration (which is included for reference). An internal resonance is discovered giving a new explanation to the practically observed asymmetry of the vertical displacement. Application of the theory to describe the reaction force induced to a suspension string is indicated.

\section{Introduction}

In the present paper we will present a study of non-linear free vibrations of suspended elastic cables.

The problem was motivated by research on an aero-elastic instability of overhead transmission lines, called galloping. This galloping is generated by a combination of wind and ice rain and results in a slow almost vertical periodic cable motion. For high enough amplitudes neighbouring conductors may touch each other, causing a short circuit and structural damage to the cables [10]. Although recognised and studied for more than fifty years, the problem is far from being solved. For example, it appears to be still not possible to design the system of towers and suspended electricity cables, possibly equipped with dampers, to be free of galloping.

An important observation is that galloping is a motion of the cable very close to a free vibration, since the forces (wind) are only small. Various aspects of galloping are therefore inherent to the free motion the cable is close to, and the study of the dynamics of free vibrating suspended cables is essential for understanding galloping. For example, the coupling between harmonics, the relation between tension and displacement, and the existence of internal resonances can be found by studying the equivalent free vibration. 
Although the related theory of tensed strings is a classic and well established part of theoretical mechanics [5], the theory of a vibrating heavy elastic suspended cable is relatively new. A number of investigations on the problem have been published for the geometry of a single span, i.e., with fixed ends $[1,2,3,4,7,9]$. However, the geometry we will mainly consider, which is the most relevant in overhead transmission line practice, consists of a series of coupled spans, and to our knowledge this has not yet been treated in the literature. Nevertheless, our theory is equally well applicable to the single span configuration, and this will therefore be included for reference.

The analysis will consist of three parts. First, we establish the model adopted, with differential equations and boundary conditions. This is relatively standard. Then we derive an asymptotically approximate problem by introducing a small parameter based on assumptions on sag, instationary amplitude, and transversal and longitudinal wave length, which are essentially the same assumptions necessary for the well-known parabola approximation of the stationary solution. The resulting (still nonlinear) problem is similar or nearly similar to that of other studies. We believe, however, that our approach is more systematic and consistent. Finally, we solve the equations by a variant of the Lindstedt-Poincaré technique [6]. For this we assume the existence of a periodic nearly harmonic solution, and expand the dependent variables in a perturbation amplitude power series (on practical grounds restricted here to three terms). The full solution includes a variety of standing and propagating waves, and some additional conditions of symmetry and regularity are applied to define the solution further.

In addition, the reaction force in the suspension string, relevant to measurements of galloping, is briefly discussed.

\section{Model}

\subsection{Differential equations and boundary conditions}

Consider a cable, fixed at the outer ends, and divided into $N$ equal spans by $N-1$ supports. These supports are inextensible suspension strings of length $a$ and negligible weight, suspended from fixed pivots separated by a distance $S$, the span size. The suspension string allows the span end to describe a circle of radius $a$, and thus provides a coupling between adjacent spans. Through all spans the cable properties are the same. The cable is linearly elastic, with negligible bending stiffness and friction effects, of uniform undeformed cross-sectional area $A$, mass per unit length $m$, and Young's modulus $E$, and with a length per span $L$ when the cable is free of tension. We parametrise the position along the cable (per span) by the variable $\ell \in[0, L]$, such that this is just the arc length when the cable is unstretched. The time variable is $\bar{t}$. The cable moves in a vertical plane provided with a Cartesian coordinate system orientated such that the gravity vector points into the negative " $y$ "-direction. The cable's position is 
$(\bar{x}(\ell, \bar{t}), \bar{y}(\ell, \bar{t}))$ with a corresponding tension $\bar{\tau}(\ell, \bar{t})$.

The equations determining $\bar{x}, \bar{y}$, and $\bar{\tau}$ are Hooke's law relating stress and strain, and Newton's law applied to the condition of equilibrium of internal and external forces on a cable element. The finally resulting equations are

$$
\begin{gathered}
\frac{\partial}{\partial \ell}\left(\frac{\bar{\tau}}{1+\bar{\tau} / E A} \frac{\partial \bar{x}}{\partial \ell}\right)=m \frac{\partial^{2} \bar{x}}{\partial \bar{t}^{2}} \\
\frac{\partial}{\partial \ell}\left(\frac{\bar{\tau}}{1+\bar{\tau} / E A} \frac{\partial \bar{y}}{\partial \ell}\right)=m g+m \frac{\partial^{2} \bar{y}}{\partial \bar{t}^{2}} \\
\left(\frac{\partial \bar{x}}{\partial \ell}\right)^{2}+\left(\frac{\partial \bar{y}}{\partial \ell}\right)^{2}=\left(1+\frac{\bar{\tau}}{E A}\right)^{2}
\end{gathered}
$$

while it may be noted that

$$
\frac{\partial \bar{x}}{\partial \ell}=(1+\bar{\tau} / E A) \cos \psi, \quad \frac{\partial \bar{y}}{\partial \ell}=(1+\bar{\tau} / E A) \sin \psi .
$$

$g$ denotes the gravity acceleration, and $\psi$ the angle between cable tangent and the horizontal. The boundary and coupling conditions are at

(i) rigid supports

$$
\begin{array}{ll}
\bar{x}=0, \bar{y}=0 & (\ell=0) \\
\bar{x}=S, \bar{y}=0 & (\ell=L)
\end{array}
$$

(ii) suspension strings

$$
\begin{aligned}
\bar{x}^{2}+(\bar{y}-a)^{2} & =a^{2} & & (\ell=0) \\
(\bar{x}-S)^{2}+(\bar{y}-a)^{2} & =a^{2} & & (\ell=L)
\end{aligned}
$$

The brackets $[\cdot]_{ \pm}$denote the difference between the value of the quantity at the right and the left side of the supports; $\phi$ is the angle of the suspension string with the vertical. The force necessary to maintain the string end at its position is usefully split up into two orthogonal components. One, tangential to the circle described by the string end, cannot be sustained by the string and has to vanish, resulting into equation (3.7). The other, however, is directed along the string, and induces the reaction force

$$
F_{s s}=[\bar{\tau} \sin (\phi-\psi)]_{ \pm} .
$$




\subsection{Small parameters and modes of vibration}

The type of motion we are interested in is further specified by

- The ratio of sag $D$ and cable length $L$ is small (typically $1 / 30$ ), so

$$
\varepsilon=D / L \rightarrow 0
$$

- The vertical displacement is of the order of the sag, so

$$
\bar{y} / L=O(\varepsilon)
$$

- The transversal wave length $\lambda_{T}$ is of the order of $L$, so

$$
\lambda_{T} / L=O(1)
$$

- The longitudinal wave length ("sound") $\lambda_{L}$ is large compared to $L$. Consistent with practice is the estimate

$$
\lambda_{L} / L=O(1 / \varepsilon)
$$

- The string length $a$ is of the order of the sag, so

$$
a / L=O(\varepsilon)
$$

Furthermore, we assume a nearly harmonic vibration, with a single dominating frequency $\bar{\omega}$. Higher harmonics not generated by the first ( $\sim$ eigensolutions) will be excluded. We will only investigate the effect of non-trivial coupling. For example, a sequence of single span solutions in phase, although a valid solution, will not be considered for the coupled configuration.

\section{Asymptotic analysis}

\subsection{Reduced problem}

The basic small parameter $\varepsilon$ will be utilised to reduce the above general problem to the asymptotically leading order problem, i.e., in terms of dimensionless variables of $O(1)$, independent of $\varepsilon$.

Since the longitudinal wave speed is $c_{L}=(E A / m)^{\frac{1}{2}}$, we have $\lambda_{L} / L=c_{L} / \bar{\omega} L=O(1 / \varepsilon)$, so the scaled frequency and corresponding time variable are given by

$$
\bar{\omega}=\omega \varepsilon(E A / m)^{\frac{1}{2}} / L, \quad \bar{t}=t L(m / E A)^{\frac{1}{2}} / \varepsilon .
$$

The transversal wave velocity is $c_{T}=(\vec{\tau} / m)^{\frac{1}{2}}$, so $\lambda_{T} / L=c_{T} / \bar{\omega} L=O(1)$. This yields for the spatial coordinate and the tension

$$
\ell=s L
$$




$$
\bar{\tau}=\varepsilon^{2} E A T
$$

Since $\bar{y} / L=O(\varepsilon)$, we introduce

$$
\bar{y}=\varepsilon L Y .
$$

With the above estimates substituted in (3.3) we obtain $\bar{x}_{\ell}=1+O\left(\varepsilon^{2}\right)$, and so

$$
\bar{x}=L\left(s+\varepsilon^{2} X\right) .
$$

Finally, we investigate the role of gravity. Substitute the present results into equation (3.2) to find the term $m g L / E A \varepsilon^{3}$ next to terms of $O(1)$. So it has to be $O(1)$ or smaller. Suppose it is small, then the stationary solution would be to leading order $Y \equiv 0$, so $D=0$, which is contradictory to our assumptions. So the term is $O(1)$ and we introduce

$$
\mu=m g L / 8 E A \varepsilon^{3}=O(1) .
$$

Since we will only consider the $\varepsilon$-approximation to leading order, we may as well assume $\omega, T, Y$, and $X$ to be $\varepsilon$-independent with an (a priori) relative error of $O\left(\varepsilon^{2}\right)$.

The basic equations (3.1-3) are then reduced to

$$
\frac{\partial}{\partial s} T=0, \quad \frac{\partial}{\partial s}\left(T \frac{\partial}{\partial s} Y\right)=8 \mu+\frac{\partial^{2}}{\partial t^{2}} Y, \quad \frac{\partial}{\partial s} X+\frac{1}{2}\left(\frac{\partial}{\partial s} Y\right)^{2}=T
$$

It is convenient to split up the solution into a stationary and instationary part:

$$
X=X_{0}+x, Y=Y_{0}+y, T=T_{0}+\tau,
$$

with boundary conditions for the stationary part $Y_{0}(0)=Y_{0}(1)=0, Y_{0}\left(\frac{1}{2}\right)=-1$, $X_{0}(0)=0, X_{0}(1)=S_{0}$, where $S_{0}$ is given by $S=L\left(1+\varepsilon^{2} S_{0}+O\left(\varepsilon^{4}\right)\right)$. The stationary solution is the well-known parabola shape

$$
Y_{0}=-4\left(s-s^{2}\right), \quad T_{0}=\mu, \quad X_{0}=\mu-\frac{4}{3}\left(1+(2 s-1)^{3}\right), \quad S_{0}=\mu-\frac{8}{3} .
$$

If we substitute (4.2) into (4.1) we obtain our fundamental instationary problem

$$
\begin{gathered}
\frac{\partial \tau}{\partial s}=0 \\
(\mu+\tau) \frac{\partial^{2} y}{\partial s^{2}}+8 \tau=\frac{\partial^{2} y}{\partial t^{2}} \\
\frac{\partial x}{\partial s}+4(2 s-1) \frac{\partial y}{\partial s}+\frac{1}{2}\left(\frac{\partial y}{\partial s}\right)^{2}=\tau
\end{gathered}
$$


The boundary conditions at rigid supports follow readily:

$$
x(0)=x(1)=y(0)=y(1)=0
$$

For the conditions at the suspension string we observe that $a \phi / L=O\left(\varepsilon^{2}\right)$ as it is of the order of the $x$-variations, so $\phi=O(\varepsilon)$, and we obtain the conditions

$$
y(0)=y(1)=0, \quad[x]_{ \pm}=0, \quad[\tau]_{ \pm}=0 .
$$

An immediate consequence of (4.3) and (4.7) is that $\tau$ is constant in $s$ and the same in all spans. This is of course only true if $N=O(1)$, since otherwise a small deflection from the constant would accumulate on a larger scale. Another way to portray this is by considering, in a matched expansion terminology, the present $O(1)$ domain as the inner region of the longitudinal wave regime of $O(1 / \varepsilon)$.

Finally, the reaction force $(3.8)$ is (to a relative error of $O\left(\varepsilon^{2}\right)$ )

$$
F_{s s}=-E A \varepsilon^{3} T\left[\frac{\partial Y}{\partial s}\right]_{ \pm}=m g L+E A \varepsilon^{3} f_{s s}
$$

with $f_{s s}=8 \tau-(\mu+\tau)[y]_{ \pm}$. The constant $m g L$ is just the weight of a span.

\subsection{Amplitude power series expansion}

The Lindstedt-Poincaré technique [6] involves the assumption of a periodic solution with fundamental frequency $\omega$, the introduction of the amplitude of the linearised solution as a small parameter $\delta$, and then expanding the full solution into a $\delta$-power series, starting with $O(\delta)$. Of course, we could have included the foregoing stationary solution (4.2) as the first, $O(1)$, term in this series, but this stationary part is so important in itself that we have taken it apart.

Since $\omega$ will also depend on $\delta$ it is convenient to introduce $t^{\prime}=\omega t$. Now we assume

$$
y=\delta y_{1}+\delta^{2} y_{2}+\delta^{3} y_{3}+\cdots
$$

and similarly for $x$ and $\tau$, and

$$
\omega=\omega_{0}+\delta^{2} \omega_{2}+\cdots
$$

Note that $\omega_{1}=0$ since $\omega$ should not depend on the sign of $\delta$. Introduce the notation $' \equiv \partial / \partial s,{ }^{\prime} \equiv \partial / \partial t^{\prime}$. The equations that result from substitution of $(4.9,10)$ into (4.3-5) and collecting like powers of $\delta$ are then

$$
\left\{\begin{array}{r}
\mu y_{1}^{\prime \prime}+8 \tau_{1}-\omega_{0}^{2} \ddot{y}_{1}=0 \\
x_{1}^{\prime}+4(2 s-1) y_{1}^{\prime}=\tau_{1}
\end{array}\right.
$$




$$
\begin{gathered}
\left\{\begin{array}{r}
\mu y_{2}^{\prime \prime}+8 \tau_{2}+\tau_{1} y_{1}^{\prime \prime}-\omega_{0}^{2} \ddot{y_{2}}=0 \\
x_{2}^{\prime}+4(2 s-1) y_{2}^{\prime}+\frac{1}{2}\left(y_{1}^{\prime}\right)^{2}=\tau_{2}
\end{array}\right. \\
\left\{\begin{array}{r}
\mu y_{3}^{\prime \prime}+8 \tau_{3}+\tau_{2} y_{1}^{\prime \prime}+\tau_{1} y_{2}^{\prime \prime}-\omega_{0}^{2} \ddot{y}_{3}-2 \omega_{0} \omega_{2} \ddot{y}_{1}=0 \\
x_{3}^{\prime}+4(2 s-1) y_{3}^{\prime}+y_{1}^{\prime} y_{2}^{\prime}=\tau_{3}
\end{array}\right.
\end{gathered}
$$

with $\tau_{1}, \tau_{2}, \tau_{3}, \cdots$ constant in $s$. The interesting solutions here are based on the harmonic solutions of the linearised problem, which generate via the nonlinear coupling sub- and super harmonics in the higher order terms. So we put

$$
y_{1}=y_{11} \sin \left(t^{\prime}\right), \quad y_{2}=y_{20}+y_{22} \cos \left(2 t^{\prime}\right), \quad y_{3}=y_{31} \sin \left(t^{\prime}\right)+y_{33} \sin \left(3 t^{\prime}\right)
$$

and similarly for $x$ and $\tau$. Substitution of (4.14) into (4.11-13) and collecting the harmonics yields

$$
\left\{\begin{aligned}
\mu y_{11}^{\prime \prime}+8 \tau_{11}+\omega_{0}^{2} y_{11} & =0 \\
x_{11}^{\prime}+4(2 s-1) y_{11}^{\prime} & =\tau_{11}
\end{aligned}\right.
$$

$$
\left\{\begin{aligned}
\mu y_{20}^{\prime \prime}+8 \tau_{20}+\frac{1}{2} \tau_{11} y_{11}^{\prime \prime} & =0 \\
x_{20}^{\prime}+4(2 s-1) y_{20}^{\prime}+\frac{1}{4}\left(y_{11}^{\prime}\right)^{2} & =\tau_{20}
\end{aligned}\right.
$$

$$
\left\{\begin{aligned}
\mu y_{22}^{\prime \prime}+8 \tau_{22}-\frac{1}{2} \tau_{11} y_{11}^{\prime \prime}+4 \omega_{0}^{2} y_{22} & =0 \\
x_{22}^{\prime}+4(2 s-1) y_{22}^{\prime}-\frac{1}{4}\left(y_{11}^{\prime}\right)^{2} & =\tau_{22}
\end{aligned}\right.
$$

$$
\left\{\begin{aligned}
\mu y_{31}^{\prime \prime}+8 \tau_{31}+\omega_{0}^{2} y_{31}+\left(\tau_{20}-\frac{1}{2} \tau_{22}\right) y_{11}^{\prime \prime}+\tau_{11}\left(y_{20}^{\prime \prime}-\frac{1}{2} y_{22}^{\prime \prime}\right)+2 \omega_{0} \omega_{2} y_{11} & =0 \\
x_{31}^{\prime}+4(2 s-1) y_{31}^{\prime}+y_{11}^{\prime}\left(y_{20}^{\prime}-\frac{1}{2} y_{22}^{\prime}\right) & =\tau_{31}
\end{aligned}\right.
$$

$$
\left\{\begin{aligned}
\mu y_{33}^{\prime \prime}+8 \tau_{33}+9 \omega_{0}^{2} y_{33}+\frac{1}{2} \tau_{22} y_{11}^{\prime \prime}+\frac{1}{2} \tau_{11} y_{22}^{\prime \prime}=0 \\
x_{33}^{\prime}+4(2 s-1) y_{33}^{\prime}+\frac{1}{2} y_{11}^{\prime} y_{22}^{\prime}=\tau_{33}
\end{aligned}\right.
$$

It may be noted that in the usual formulation of the Lindstedt-Poincare technique $\omega_{2}$ is determined by the condition of vanishing secular terms. Here we have simplified this to the condition that there exists a solution of the present type. 


\section{Solution}

\subsection{Coupled spans}

A most important property of vibrating coupled spans is the fact that to leading order the tension vanishes and contains no first harmonic. This makes the motion of a coupled span considerably different from the symmetric modes of a single span. In a coupled span the restoring force is basically gravity, without tension variation to leading order ( $\sim$ first harmonic), while in a single span symmetric mode both gravity and elasticity are equally important. The respective motion may therefore be called: gravity and elasto-gravity waves.

By standard techniques we find for the $i$-th span $(1 \leq i \leq N)$ the general solution

$$
\begin{aligned}
& \tau_{11}=0, \quad y_{11}=A_{i} \sin (k s), \quad \text { where } \quad k=\omega_{0} / \sqrt{\mu}=(2 n+1) \pi \\
& x_{11}=B_{i}-4(2 s-1) A_{i} \sin (k s)-8 A_{i} \cos (k s) / k \\
& B_{i}=8\left(A_{i}+2\left(A_{1}+A_{2}+\cdots+A_{i-1}\right)\right) / k \\
& \tau_{20}=\frac{3}{8} \omega_{0}^{2} \overline{A^{2}} /(16+3 \mu), \quad y_{20}=4 \tau_{20}\left(s-s^{2}\right) / \mu \\
& x_{20}=C_{i}+\tau_{20} s+\frac{8}{3} \tau_{20}\left(1+(2 s-1)^{3}\right) / \mu-\frac{1}{8} k^{2} A_{i}^{2}(s+\sin (2 k s) / 2 k) \\
& C_{i}=(i-1) \tau_{20}(1+16 / 3 \mu)-\frac{1}{8} k^{2}\left(A_{1}^{2}+\cdots+A_{i-1}^{2}\right) \\
& x_{22}=D_{i}+\tau_{22} s-8 \tau_{22}(1+(2 s-1) \cos (2 k s)-\sin (2 k s) / k) / \omega_{0}^{2}+\frac{1}{8} k^{2} A_{i}^{2}(s+\sin (2 k s) / 2 k) \\
& D_{i}=-(i-1) \tau_{22}\left(16 / \omega_{0}^{2}-1\right)+\frac{1}{8} k^{2}\left(A_{1}^{2}+\cdots+A_{i-1}^{2}\right) \\
& \tau_{31}=0, \quad y_{31}=0 \\
& x_{31}=E_{i}+A_{i}\left(4 k \tau_{20}(2 s-1) \sin (k s)+\left(8 \tau_{20}+\tau_{22}\right) \cos (k s)+\frac{1}{3} \tau_{22} \cos (3 k s)\right) / \mu k \\
& E_{i}=-B_{i}\left(\tau_{20}+\frac{1}{6} \tau_{22}\right) / \mu \\
& \tau_{33}=0, \quad y_{33}=\frac{1}{16} \tau_{22} A_{i} \sin (k s) / \mu \\
& x_{33}=F_{i}-\tau_{22} A_{i}\left(\frac{1}{4} k(2 s-1) \sin (k s)+\frac{3}{2} \cos (k s)+\frac{1}{3} \cos (3 k s)\right) / \mu k \\
& F_{i}=\frac{11}{48} \tau_{22} B_{i} / \mu
\end{aligned}
$$

where $\overline{A^{2}}=\left(A_{1}^{2}+A_{2}^{2}+\cdots+A_{N}^{2}\right) / N$. The frequency shift necessary for a solution $y_{31}$ is

$$
\omega_{2}=\frac{1}{4} \omega_{0}\left(2 \tau_{20}-\tau_{22}\right) / \mu
$$


and the amplitudes $A_{i}$ are further restricted by the condition

$$
A_{1}+A_{2}+\cdots+A_{N}=0
$$

Note the breakdown of the solution if $\omega_{0}=4$ by the vanishing denominator of $\tau_{22}$. Although further research is necessary to reveal the character of this singularity, it is to be interpreted as a resonance between first and second harmonic.

Equation (5.7) does not determine a unique solution (except for $N=2$ ). Some special cases, however, are useful to be considered in detail.

A most regular solution is found if $N$ is even with

$$
A_{i}=(-1)^{i}
$$

since this is independent of $N$. As every even suspension string is now motionless, it is in fact just a sequence of $(N=2)$-solutions. Furthermore, the suspension string reaction force (4.8) in $\delta$-expanded form only depends on the tension and simplifies to $f_{s s}=8 \delta^{2} \tau_{22} \cos \left(2 t^{\prime}\right)+O\left(\delta^{4}\right)$, since by symmetry $\left[y_{11}^{\prime}\right]_{ \pm}=\left[y_{33}^{\prime}\right]_{ \pm}=0$, and inertial effects are absent.

If $N$ is odd, we cannot have all $A_{i}$ equal in magnitude, but an almost uniform middle region $2 \leq i \leq N-1$ is obtained with $A_{1}=A_{N}=-\frac{1}{2}, \quad A_{i}=(-1)^{i} \quad(2 \leq i \leq N-1)$.

\subsection{Single span}

The asymmetric modes of a single span are very similar to the periodic solution (5.8) for an even number of coupled spans. The only difference is the wave number $k$. Instead of an odd it is now an even multiple of $\pi: k=2 n \pi$.

The symmetric modes are considerably different, and not reported to occur often with galloping. However, it is the configuration considered most in the literature, so we will, for reference, briefly present it here in the context of our analysis.

It is convenient to introduce $\hat{\tau}=\tau / \mu$, and $z=s-\frac{1}{2}$ where $\tau$ is any $\tau_{11}, \cdots$. The solution is then

$$
\hat{\tau}_{11}=\frac{1}{8} k^{2} \cos \left(\frac{1}{2} k\right), \quad y_{11}=\cos (k z)-\cos \left(\frac{1}{2} k\right)
$$

where $k=\omega_{0} / \sqrt{\mu}$ is a solution of the equation $\tan \left(\frac{1}{2} k\right)=\frac{1}{2} k\left(1-\frac{1}{64} \omega_{0}^{2}\right)$

$$
\begin{gathered}
x_{11}=\hat{\tau}_{11} \mu z+8(\sin (k z)-k z \cos (k z)) / k \\
\hat{\tau}_{20}=\frac{3}{8} k^{2}(3 \sin (k) / k-2 \cos (k)-1) /(16+3 \mu) \\
y_{20}=-\frac{1}{2} \hat{\tau}_{11}\left(\cos (k z)-\cos \left(\frac{1}{2} k\right)\right)-\hat{\tau}_{20}\left(4 z^{2}-1\right)
\end{gathered}
$$




$$
x_{20}=\hat{\tau}_{20} \mu z+\frac{64}{3} \hat{\tau}_{20} z^{3}-\frac{1}{2} k \cos \left(\frac{1}{2} k\right)(\sin (k z)-k z \cos (k z))+\frac{1}{16} k(\sin (2 k z)-2 k z)
$$

$$
\begin{gathered}
\hat{\tau}_{22}=\frac{1}{24} k^{3}(5 \sin (k)-2 \tan (k)-3 k) /\left(\omega_{0}^{2}-16+16 \tan (k) / k\right) \\
y_{22}=2\left(\hat{\tau}_{22}+\frac{2}{3} \hat{\tau}_{11}^{2}\right)(\cos (2 k z)-\cos (k)) / k^{2} \cos (k)-\frac{1}{6} \hat{\tau}_{11}\left(\cos (k z)-\cos \left(\frac{1}{2} k\right)\right) \\
x_{22}=\hat{\tau}_{22} \mu z-\frac{4}{3} \hat{\tau}_{11}(\sin (k z)-k z \cos (k z)) / k-\frac{1}{16} k(\sin (2 k z)-2 k z) \\
+8\left(\hat{\tau}_{22}+\frac{2}{3} \hat{\tau}_{11}^{2}\right)(\sin (2 k z)-2 k z \cos (2 k z)) / k^{3} \cos (k)
\end{gathered}
$$

and a frequency shift of

$$
\begin{gathered}
\omega_{2}=\frac{2}{3} \omega_{0} \hat{\tau}_{11} \cos \left(\frac{1}{2} k\right)\left[\left(5 \hat{\tau}_{22}+18 \hat{\tau}_{20}+\frac{19}{3} \hat{\tau}_{11}^{2}\right)\left(2 \tan \left(\frac{1}{2} k\right)-k\right)\right. \\
\left.-\left(3 \hat{\tau}_{22}-6 \hat{\tau}_{20}+5 \hat{\tau}_{11}^{2}\right) k \tan ^{2}\left(\frac{1}{2} k\right)+4\left(\hat{\tau}_{22}+\frac{2}{3} \hat{\tau}_{11}^{2}\right)\left(\tan \left(\frac{1}{2} k\right)-\tan (k)+\frac{1}{2} k\right)\right] \\
/ k^{3}(2+\cos (k)-3 \sin (k) / k)
\end{gathered}
$$

The other third order terms become increasingly lengthy and complex, and can be found in [8]. The linear solution (5.9) is similar to what is presented in for example $[2,3,9]$. The higher order corrections are new. Nonlinear extensions to the linear theory have been described in $[2,4,7]$, but for an equation in spatially averaged variables using an assumed shape function.

\section{Discussion and examples}

A most interesting result of the present analysis for coupled spans is the resonance frequency $\omega_{0}=4$, where the higher order terms become comparable to the first, and the solution breaks down. When $\omega_{0}$ is not too far from 4 , the singular denominator easily amplifies the second harmonic enough to render the displacement $y$ asymmetrically upward or downward, depending on the sign of $\omega_{0}-4$ (i.e., the driven second harmonic is in or out of phase with its source, the first harmonic). The common situation for coupled spans of transmission lines seems to be a relative elasticity $\mu$ smaller than $16 / \pi^{2}=1.62$, and so a frequency $\omega_{0}$ smaller than 4 , resulting into an (indeed reported) asymmetry upwards. However, by only changing the parameters a little we might as well have an asymmetry downwards. This is completely different from the always upward asymmetry of a single span symmetric mode, which is usually a smaller effect and has a displacement maximum always accompanied by a tension minimum.

We have plotted examples of a coupled multiple and a single span configuration. The vertical displacement $d Y$ at $s=\frac{1}{2}$ and tension variation $d T$ are shown for a cable, given by $S=300 \mathrm{~m}, m g=10 \mathrm{~N} / \mathrm{m}, E A=15.10^{6} \mathrm{~N}, D=9 \mathrm{~m}$ with $\varepsilon=0.03$ and $\mu=0.93$. In figure 1 we have a 2 -span (eq.(5.8)), with $\omega_{0}=3.03$, and in figure 2 a single span, with $\omega_{0}=7.22$. Absence of the first harmonic in the 2-span tension, presence of other harmonics, asymmetry in $d Y$, etc., are clear. 


\section{Acknowledgement}

This paper is based on a study, prepared at the Mathematical Consulting Department, University of Nijmegen, under contract for the NV Provinciale Limburgse Elektriciteits Maatschappij PLEM, Maastricht. The support, interest, and stimulating discussions of (in alphabetical order) A.H.P. van der Burgh, P.H.Leppers, and J.Molenaar are gratefully acknowledged.

\section{References}

[1] A.H.P. van der Burgh, An asymptotic theory for the free vibrations of an iced two-conductor bundled transmission line, Asymptotic Analysis II, Lecture Notes in Mathematics 985, Springer-Verlag, Berlin, 413-430, 1983.

[2] P. Hagedorn and B. Schäfer, On non-linear free vibrations of an elastic cable, International Journal of Non-linear Mechanics, 15, 333-340, 1980.

[3] H.M. Irvine and T.K. Caughey, The linear theory of free vibrations of a suspended cable, Proceedings of the Royal Society of London A341, 299-315, 1974.

[4] A. Luongo, G. Rega, and F. Vestroni, Planar non-linear free vibrations of an elastic cable, International Journal of Non-linear Mechanics, 19(1), 39-52, 1984.

[5] P.M. Morse and H. Feshbach, Methods of Theoretical Physics, Mc Graw-Hill, New York, 1953.

[6] A.H. Nayfeh and D.T. Mook, Nonlinear Oscillations, John Wiley \& Sons, New York, 1979

[7] G. Rega, F. Vestroni, and F. Benedettini, Parametric analysis of large amplitude free vibrations of a suspended cable, International Journal of Solids and Structures 20(2), 95-105, 1984.

[8] S.W. Rienstra, A nonlinear theory of free vibrations of single and coupled suspended elastic cables, Report WD 88-06, Katholieke Universiteit Nijmegen, The Netherlands, 1988

[9] A. Simpson, On the oscillatory motions of translating elastic cables, Journal of Sound and Vibration, 20(2), 177-189, 1972.

[10] A. Simpson, Wind-induced vibration of overhead power transmission lines, Sci. Prog. Oxford 68, 285-308, 1983.

S.W. Rienstra, Department of Mathematics and Computing Science, University of Technology, P.O.box 513, 5600 MB Eindhoven, The Netherlands 


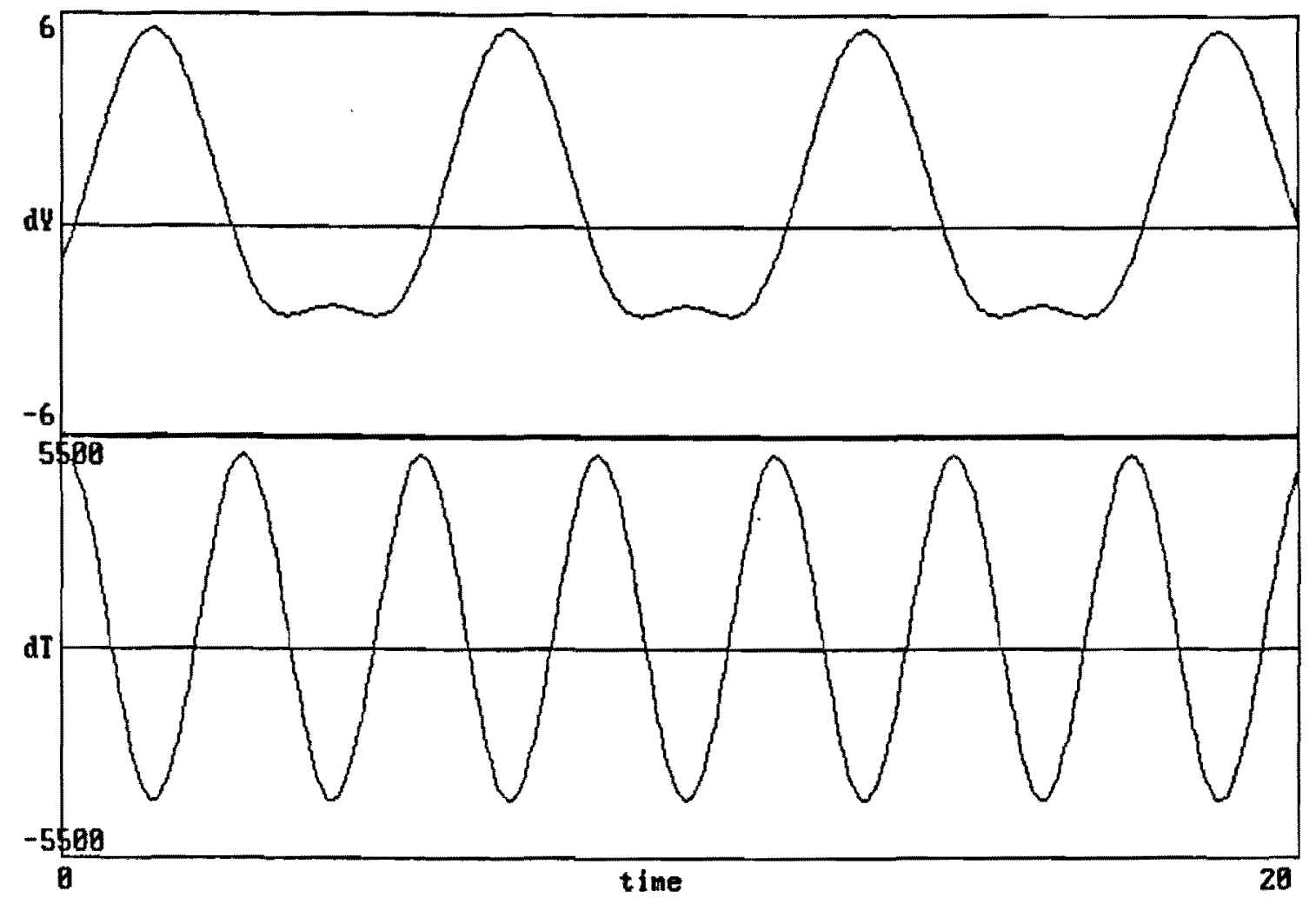

Figure 1. 2 span in 1 st mode at $s=\frac{1}{2} \quad(\delta=.45$, frequency=.1731 $)$

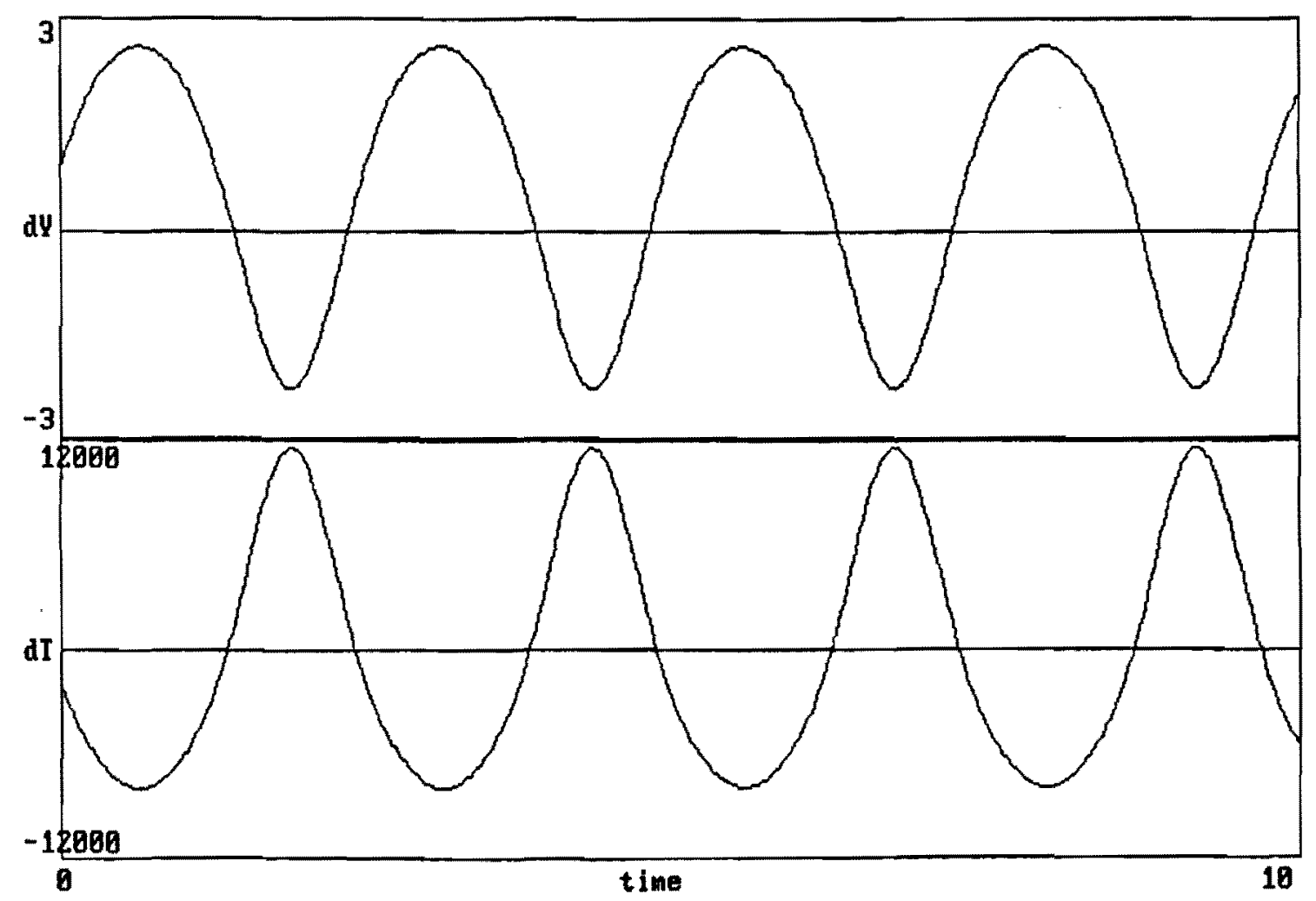

Figure 2. 1 span in 1st mode at $s=\frac{1}{2} \quad(\delta=.13$, frequency $=.4081)$ 